\section{Asymptotically Efficient Estimation of Spectral Moments}

José M. B. Dias and José M. N. Leitão

\begin{abstract}
This correspondence studies parametric estimation of spectral moments of a zero-mean complex Gaussian stationary process immersed in independent Gaussian noise. With the merit of the maximumlikelihood (ML) approach as motivation, this work exploits a Whittle's type objective function that is able to capture the relevant features of the log-likelihood function while being much more manageable. The resulting estimates are strongly consistent and asymptotically efficient. As an example, application to Doppler weather radar data is considered.
\end{abstract}

\section{INTRODUCTION}

In general, spectral estimation addresses the problem of power spectral density (PSD) inference from a finite observation of the underlying process. There are situations, however, in which the goal is the determination of PSD functionals, rather than the PSD itself; a relevant example is spectral moments (SM) estimation, which is the main theme of this correspondence. The motivation stems from our previous work on Doppler weather radar [1]-[3], where the goal is the determination of the three first SM within the so-called resolution volume [4]. These moments are closely related to physical entities of interest: the zeroth moment (mean power) is related to the water content, the first moment (mean frequency) is related to the hydrometeors mean radial velocity, and the square root of the second centered moment (spectral width) is a measure of the hydrometeor's velocity dispersion. Besides Doppler weather radar, SM estimation finds application on clear-air turbulence measurement, ultrasound imaging in medicine, and synthetic aperture radar, to name a few.

Well-known nonparametric SM estimation techniques are the pulse pair (PP) [5] and the periodogram-based (PB) estimate [4]. For bandlimited processes, exact ML nonparametric estimates were derived in [2].

Concerning parametric approaches, the optimality characteristics of the ML criterion (at least asymptotically) have fostered its study in several fields. However, determining the ML solution is often cumbersome (both in analytical and computational senses) [6]. As a consequence, the ML criterion has been put aside where SM is concerned.

Our objective in this work is to come out with a parametric SM estimator that asymptotically exhibits ML properties having a tolerable complexity. This is attained by replacing the log-likelihood function with a close Whittle's-type objective function [7]. By hypothesis, the parametric model is known up to a multiplicative constant, a frequency shift, and a scale factor.

The main contributions presented in this work are the following:

a) a criterion yielding asymptotically efficient SM estimates

b) a result stating the strong consistency of the proposed estimates

c) an approximate expression for the estimate's bias

d) a procedure of moderate complexity providing the SM estimates.

Manuscript received April 17, 1993; revised February 8, 1995. The associate editor coordinating the review of this paper and approving it for publication was Prof. Douglas Williams.

The authors are with the Instituto de Telecomunicações and Departamento de Engenharia Electrotécnica e de Computadores, Instituto Superior Técnico, Lisbon, Portugal.

IEEE Log Number 9413303.
The paper is organized as follows: Section II introduces notation and hypotheses about the underlying process, formulates the problem, and derives the approximate ML estimator; statistical characterization is also provided. Section III specializes the previous concepts to Doppler-spread data. Section IV presents an application using weather radar data.

\section{Problem Formulation and ApProximate ML Estimator}

Let $\mathcal{X}=\{x(t), t \in \Re\}$ and $\mathcal{N}=\{n(t), t \in \Re\}$ be independent zero-mean complex Gaussian strictly stationary processes, with covariance functions (CF's) $R_{x}(\tau)$ and $R_{n}(\tau)$, respectively. The PSD associated with $R_{x}(\tau)$ is denoted by $S_{x}(f)$. We assume that $R_{n}(\tau)$ is known in advance (notice that, in many applications, $R_{n}(\tau)$ can be measured/estimated with arbitrary precision). Since our approach is parametric, we write $R_{x}(\tau, \theta)$ and $S_{x}(f, \theta)$ with $\theta \in \Theta \subset \Re^{p}$ and $p \geq 1$.

The problem of SM estimation is then stated as follows: given $M$ consecutive samples $\mathbf{Y}=\left[Y_{1}, \cdots, Y_{M}\right]^{T}$, with $Y_{i}=y\left(i T_{s}\right)=$ $x\left(i T_{s}\right)+n\left(i T_{s}\right)$, design estimators of the spectral moments (which are assumed to exist) given by

$$
\begin{aligned}
m_{k}\left(S_{x}\right) & \equiv \int_{-\infty}^{\infty} f^{k} S_{x}(f, \theta) d f \\
& =\frac{1}{(2 \pi j)^{k}} R_{x}^{(k)}(0, \theta), \quad k=0,1,2, \cdots
\end{aligned}
$$

where $(j \equiv \sqrt{-1})$ and $R_{x}^{(k)}(0, \theta)$ stands for the $k$ th derivative of $R_{x}(0, \theta)$ with respect to $\tau$, at $\tau=0$. Besides $m_{k}$, we are also interested in the spectral width

$$
\sigma\left(S_{x}\right)=\left[\frac{m_{2}}{m_{0}}-\left(\frac{m_{1}}{m_{0}}\right)^{2}\right]^{1 / 2} .
$$

Let $\hat{\theta}^{\mathrm{ml}}$ be the ML estimate of parameter $\theta$. The invariance of ML estimation [8] implies that $\hat{m}_{k}^{\mathrm{ml}}=m_{k}\left[S_{x}\left(f, \hat{\theta}^{\mathrm{ml}}\right)\right]$ and that $\hat{\sigma}^{\mathrm{ml}}=\sigma\left[S_{x}\left(f, \hat{\theta}^{\mathrm{ml}}\right)\right]$, i.e., ML estimation of $\mathrm{SM}$ reduces to $\mathrm{ML}$ estimation of parameter $\theta$. Therefore, we will focus our attention on $\hat{\theta}^{\mathrm{ml}}$.

Define $\mathbf{R}_{y}(\theta) \equiv E\left[\mathbf{Y} \mathbf{Y}^{H}\right]=\left\{R_{y}\left[(i-j) T_{s}, \theta\right], i, j=1, \cdots, M\right\}$ (where $(\cdot)^{H}$ stands for transpose conjugate), with $R_{y}(\tau, \theta)=$ $R_{x}(\tau, \theta)+R_{n}(\tau)$. Recalling that $\mathcal{X}$ and $\mathcal{N}$ are zero-mean complex Gaussian strictly stationary processes and assuming that $\mathbf{R}_{y}^{-1}(\theta)$ exists for $\theta \in \Theta$, it follows that the probability density function of $\mathbf{Y}$ is

$$
f_{y}(\mathbf{Y}=\mathbf{y} \mid \theta)=\frac{1}{\pi^{M}\left|R_{y}(\theta)\right|} \exp \left[-\mathbf{y}^{H} \mathbf{R}_{y}^{-1}(\theta) \mathbf{y}\right] .
$$

Let $\theta_{0}$ be the true parameter; its ML estimate is given by

$$
\hat{\theta}^{\mathrm{ml}}=\arg \max _{\theta \in \Theta} \Lambda(\mathbf{Y} \mid \theta) \text { where } \Lambda(\mathbf{Y} \mid \theta)=\ln \left[f_{y}(\mathbf{Y} \mid \theta)\right] .
$$

Finding $\hat{\theta}^{\mathrm{ml}}$ demands explicit expressions for $\left|\mathbf{R}_{y}(\theta)\right|$ and $\mathbf{R}_{y}^{-1}(\theta)$. In most cases, these expressions are very difficult to obtain [6]. It is thus natural to apply iterative schemes to compute $\hat{\theta}^{\mathrm{ml}}$; relevant examples are the estimation of structured covariance matrices [9], [10] and the estimation of ARMA parameters [11]; the expectation maximization algorithm has also been applied to iteratively solve 
ML maximization problems [12]. Regarding SM, the computational burden of these methods is unbearable in most practical applications. Seeking for an estimator with ML (or nearly ML) features but with lighter complexity, we exploit an objective function $L(\mathbf{Y} \mid \theta)$ having the same structure of $\Lambda(\mathbf{Y} \mid \theta)$ but where $\mathbf{R}_{y}(\theta)$ is replaced with $\mathbf{R}_{c y}(\theta)=\left\{R_{c y}\left[(i-j) T_{s}, \theta\right], i, j=1, \cdots, M\right\}$, and $R_{c y}(\tau, \theta)=$ $\sum_{k=-\infty}^{\infty} R_{y}\left(\tau+k M T_{s}, \theta\right)$. Assuming that $R_{y}\left(i T_{s}, \theta\right)$ is negligible for $|i| \geq m$ and $M>2 m+1$, then $R_{c y}(\tau, \theta)$ is a periodic extension of $R_{y}(\tau, \theta)$. Observe that $\mathbf{R}_{c y}(\theta)$ is a right circulant matrix; with exception of the upper right and lower left corners, matrices $\mathbf{R}_{c y}(\theta)$ and $\mathbf{R}_{y}(\theta)$ are equal. Thus, if $M \gg 2 m$, it can be expected that estimates based on $\mathbf{R}_{c y}(\theta)$ and on $\mathbf{R}_{y}(\theta)$ are close.

Consider the eigendecomposition $\mathbf{R}_{c y}(\theta)=\mathbf{F} \boldsymbol{\Sigma}_{y}(\theta) \mathbf{F}^{H}$. Since $\mathbf{R}_{c y}(\theta)$ is right circulant, the eigenvector matrix is $\mathbf{F}=$ $M^{-1 / 2}\left\{e^{-j \frac{2 \pi}{M} i k}, i, j=0, \cdots, M-1\right\}$ and the eigenvalue matrix $\Sigma_{y}(\theta)=\operatorname{diag}\left[\lambda_{0}(\theta), \ldots, \lambda_{M-1}(\theta)\right]$ is the discrete Fourier transform of the first row of $\mathbf{R}_{c y}(\theta)$ [8].

Replacing $\mathbf{R}_{y}(\theta)$ with $\mathbf{R}_{c y}(\theta)$ in $\Lambda(\mathbf{Y} \mid \theta)$, the objective function $L(\mathbf{Y} \mid \theta)$ is equivalent to

$$
\begin{aligned}
& L(\mathbf{Y} \mid \theta)= \text { const }-M^{-1}\left[\ln \left|\mathbf{R}_{c y}(\theta)\right|+\mathbf{Y}^{H} \mathbf{R}_{c y}^{-1}(\theta) \mathbf{Y}\right] \\
&=\text { const }-M^{-1}[ \ln \left|\mathbf{\Sigma}_{y}(\theta)\right|+\left\{\mathbf{F}^{H} \mathbf{Y}\right\}^{H} \\
&\left.\times \mathbf{\Sigma}_{y}^{-1}(\theta)\left\{\mathbf{F}^{H} \mathbf{Y}\right\}\right] \\
&=\text { const }-M^{-1}\left(\sum_{i=0}^{M-1} \ln \lambda_{i}(\theta)+\sum_{i=0}^{M-1} \frac{\tilde{\lambda}_{i}}{\lambda_{i}(\theta)}\right)
\end{aligned}
$$

where $\tilde{\lambda}_{i}$ is the periodogram of the observed sequence $\left\{Y_{i}\right\}_{i=1}^{i=M}$ computed at $F_{i}=i / M$ for $i=0, \cdots, M-1$, and $\lambda_{i}(\theta)=\lambda\left(F_{i}, \theta\right)$ with (we assume that $M>2 m+1$ )

$$
\begin{aligned}
\lambda(F, \theta) & =\sum_{k=0}^{M-1} R_{c y}\left(k T_{s}, \theta\right) e^{-j 2 \pi F k} \\
& =\sum_{k=-\infty}^{\infty} R_{y}\left(k T_{s}, \theta\right) e^{-j 2 \pi F k} .
\end{aligned}
$$

The last sum in (8) is, by definition, the discrete time Fourier transform (DTFT) of $R_{y}\left(k T_{s}, \theta\right)$.

The estimation criterion (4) with $\Lambda(\mathbf{Y} \mid \theta)$ replaced by $L(\mathbf{Y} \mid \theta)$ leads to an estimate herein denoted by $\hat{\theta}$ and termed the approximate maximum likelihood (AML). It corresponds to a generalization of Whittle's ML approximation method [7] and is significantly more manageable than the exact log-likelihood function while behaving (asymptotically) in the same way. Related results are found in [6]. This work proposes the application of the concepts just stated to SM estimation; this makes sense if the AML estimate $\hat{\theta}$ is a good substitute for $\hat{\theta}^{\mathrm{ml}}$. Results presented ahead provide insight into this matter.

\section{A. Asymptotic Properties}

Result 1: Let $\left\{Y_{t}\right\}$ be a strictly stationary zero-mean complex Gaussian sequence with covariance $E\left[Y_{t+k} Y_{t}^{*}\right]=R_{y}\left(k T_{s}, \theta_{0}\right)$ such that $\theta_{0}$ belongs to the compact set $\Theta$ contained in an open set $S \subset \Re^{p}$, and $\sum_{k=-\infty}^{\infty}|k|\left|R_{y}\left(k T_{s}, \theta\right)\right|^{2}<c<\infty$ for $\theta \in S$. In addition, let the partial derivatives $\partial \lambda^{-1}(F, \theta) / \partial \theta_{i}$ be continuous for all components $\theta_{i} \in \theta$ with $(F, \theta) \in[-1 / 2,1 / 2] \times S$. Assume that if $\theta_{1}, \theta_{2} \in S$ and $\theta_{1} \neq \theta_{2}$, then $\lambda\left(F, \theta_{1}\right) \neq \lambda\left(F, \theta_{2}\right)$ for almost all $F$. Under these conditions, the AML estimate $\hat{\theta}(M)$ is strongly consistent: $\hat{\theta}(M) \rightarrow \theta_{0}$ with probability one (w.p.1), as $M \rightarrow \infty$.

Result 2: Assume the hypotheses of Result 1 and that $\partial^{2} \lambda(F, \theta)$ $/\left(\partial \theta_{i} \partial \theta_{j}\right)$ is continuous for all components $\theta_{i}$ of $\theta$ with $(F, \theta) \in$ $[-1 / 2,1 / 2] \times S$. Hence, the random vector $\xi(M)=\sqrt{M}(\hat{\theta}(M)$ $\left.-\theta_{0}\right)$ is asymptotically zero-mean Gaussian with covariance matrix $\Gamma^{-1}\left(\theta_{0}\right)$, where

$$
\Gamma(\theta)=-\int_{-1 / 2}^{1 / 2} \frac{\nabla \lambda(F, \theta) \nabla^{T} \lambda(F, \theta)}{\lambda^{2}(F, \theta)} d F
$$

is the normalized asymptotic information matrix.

B. Bias

Result 3: Assume the hypotheses of Result 2 and that $\sum_{k=-\infty}^{\infty}|k|$ $\left|R_{y}\left(k T_{s}, \theta\right)\right|<\infty$ for $\theta \in S$. Under these conditions, the estimator bias verifies

$$
\begin{aligned}
& M\left\{\Gamma_{i j}\left(\theta_{0}\right)-M^{-1} \varepsilon_{i j}\left(\theta_{0}\right)\right\} E\left[\left(\hat{\theta}(M)-\theta_{0}\right)\right] \\
& \quad=\left\{\varepsilon_{i}\left(\theta_{0}\right)\right\}+O(M)
\end{aligned}
$$

with

$$
\begin{aligned}
\varepsilon_{i_{1} \cdots i_{k}}(\theta) & =\int_{-1 / 2}^{1 / 2} \beta(F, \theta) \frac{\partial^{k} \lambda^{-1}(F, \theta)}{\partial \theta_{i_{1}} \cdots \partial \theta_{i_{k}}} d F, \\
\beta(F, \theta) & =\sum_{k=-\infty}^{\infty}|k| R_{y}\left(k T_{s}, \theta\right) e^{-j 2 \pi F k}
\end{aligned}
$$

where $O(M)$ is proportional to $M^{-1}$ and $\Gamma$ is the asymptotic information matrix given by (9).

Proofs of Results 1 and 3 are carried out in [3]. Result 2 is proved in $[6$, Ch. II].

\section{Application to Doppler-Spread Targets}

We are interested in the SM of a process with $\mathrm{CF}$

$$
R_{x}(\tau, \theta)=\theta_{0} r_{x}\left(\theta_{2} \tau\right) \exp \left(j 2 \pi \theta_{1} \tau\right)
$$

where $r_{x}(0)=1, r_{x}(\tau)$ is a priori known, $\theta_{0}>0$, and $\theta_{2}>0$. The CF (13) is typical of frequency-spread targets, as it is the case in all the applications mentioned in the introduction.

For the CF (13), the objective function (7) becomes

$$
\begin{aligned}
L(\mathbf{Y} \mid \theta)= & \text { const }-M^{-1} \\
& \times\left(\sum_{i=0}^{M-1} \ln \lambda_{0}\left(F_{i}\right)+\sum_{i=0}^{M-1} \frac{\tilde{\lambda}_{i}}{\lambda_{0}\left(F_{i}-\theta_{1} T_{s}\right)}\right)
\end{aligned}
$$

where $\lambda_{0}(F)$ is the DTFT of $R_{y}\left[k T_{s},\left(\theta_{0}, 0, \theta_{2}\right)\right]$. To maximize (14), we propose the Newton-type iteration

$$
\hat{\theta}^{k+1}=\hat{\theta}^{k}+\Gamma^{-1}\left(\hat{\theta}^{k}\right) \nabla L\left(\mathbf{Y} \mid \hat{\theta}^{k}\right) .
$$

Compared with the Newton-Raphson iterative scheme, expression (15) uses matrix $-\mathbf{\Gamma}\left(\hat{\theta}^{k}\right)$ instead of $\mathbf{H}\left(\mathbf{Y} \mid \hat{\theta}^{k}\right)$, which is the Hessian of $L\left(\mathbf{Y} \mid \hat{\theta}^{k}\right)$. An informal justification for (15) is the following: Under regularity conditions as those of Result $2, \mathbf{H}\left(\mathbf{Y} \mid \theta_{0}\right)=$ $-\boldsymbol{\Gamma}\left(\theta_{0}\right)+\boldsymbol{\Delta}$, where $E\|\boldsymbol{\Delta}\|^{2}=O(M)$ [3]. Hence, for $\left\|\hat{\theta}^{k}-\theta_{0}\right\| \simeq 0$, it follows that $\mathbf{H}\left(\mathbf{Y} \mid \hat{\theta}^{k}\right) \simeq-\Gamma\left(\hat{\theta}^{k}\right)+\Delta^{\prime}$ with $E\left\|\Delta^{\prime}\right\|^{2}=O(M)$. It should be noted, however, that $\mathbf{H}\left(\mathbf{Y} \mid \hat{\theta}^{k}\right)$ may largely diverge from $\Gamma\left(\hat{\theta}^{k}\right)$ as greater displacements $\left\|\hat{\theta}^{k}-\theta_{0}\right\|$ are considered. Nevertheless, the iterative scheme (15) has shown to be much more robust than the pure Newton-Raphson. The reason is that, even for moderate displacements of $\left\|\hat{\theta}^{k}-\theta_{0}\right\|, L\left(\mathbf{Y} \mid \hat{\theta}^{k}\right)$ exhibits large deviations from the parabolic shape; it often happens that $\mathbf{H}\left(\mathbf{Y} \mid \hat{\theta}^{k}\right)$ has very small (negative) or even positive eigenvalues, whereas $\mathbf{\Gamma}\left(\hat{\theta}^{k}\right)$ keeps close to $\Gamma\left(\theta_{0}\right)$. Another advantage of (15) is that $\Gamma^{-1}\left(\hat{\theta}^{k}\right)$ can be computed off line.

The choice of the starting point $\hat{\theta}^{0}$ is crucial; notice that (14) depends on the periodogram $\tilde{\lambda}_{i}$, on which the PB estimate is built:

$$
\hat{m}_{k}^{\mathrm{pb}}=\frac{1}{M} \sum_{i=-M / 2}^{M / 2-1}\left(\frac{i f_{s}}{M}\right)^{k}\left[\tilde{\lambda}_{i}-N_{i}\right]
$$




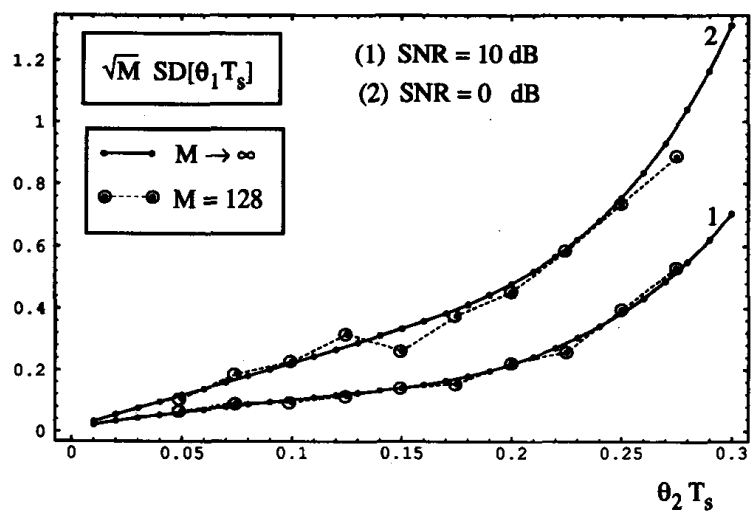

Fig. 1. Solid lines plot the normalized asymptotic CRB (standard deviation) of the first spectral moment (mean frequency). The points inside the circles show the sample standard deviation (100 Monte Carlo runs per point) of the AML estimator.

where $M$ is assumed even, and $N_{i}$ is the DTFT of $R_{n}(\tau)$. Furthermore, by applying (1) to (13), we obtain

$$
\begin{aligned}
& \theta_{0}=m_{0}, \\
& \theta_{2}=2 \pi \sigma_{x}\left(S_{x}\right)\left[-r_{r}^{(2)}(0)-\left(r_{i}^{(1)}(0)\right)^{2}\right]^{-1 / 2}, \\
& \theta_{1}=\frac{m_{1}\left(S_{x}\right)}{m_{0}\left(S_{x}\right)}-\left(\frac{\theta_{2}}{2 \pi}\right) r_{i}^{(1)}(0)
\end{aligned}
$$

where $r_{i}(\tau)$ and $r_{r}(\tau)$ are the real and imaginary parts of $r_{x}(\tau)$, respectively. Therefore, concerning the starting point $\hat{\theta}^{0}$ of the Newton-type algorithm, we proceed as follows:

a) Compute $\left(\hat{m}_{0}^{\mathrm{pb}}, \hat{m}_{1}^{\mathrm{pb}}, \hat{m}_{2}^{\mathrm{pb}}\right)$ according to (16).

b) Use (17)-(19) to obtain $\hat{\theta}^{0}=\left(\hat{\theta}_{1}^{0}, \hat{\theta}_{2}^{0}, \hat{\theta}_{3}^{0}\right)$.

\section{EXAMPLE: DOPPLER WEATHER RADAR DATA}

In this section, we take $r_{x}(\tau)=\exp \left(-2 \pi^{2} \tau^{2}\right)$ and $R_{n}\left(i T_{s}\right)$ $=N_{0} \delta(i)$. This is typical of weather radar data [13]. Hence, it follows that

$$
\begin{aligned}
\lambda_{0}(F)= & \frac{\theta_{0}}{\sqrt{2 \pi}\left(\theta_{2} T_{s}\right)} \\
& \times \sum_{k=-\infty}^{\infty} \exp \left(-\frac{1}{2}\left[\frac{1}{\left(\theta_{2} T_{s}\right)}(F-k)\right]^{2}\right)+N_{0} .
\end{aligned}
$$

In all the results presented, the sample size is $M=128$. For each value of $\theta$, the iterative scheme (15) was applied over 100 independent data sets, using the stop rule $\left|\left(\theta_{0}^{k}-\theta_{0}^{k-1}\right) / \theta_{0}^{k}\right|<$ $10^{-4},\left|\left(\theta_{1}^{k}-\theta_{1}^{k-1}\right)\right|<10^{-4} T_{s}^{-1}$, and $\left|\left(\theta_{2}^{k}-\theta_{2}^{k-1}\right) / \theta_{2}^{k}\right|<10^{-4}$. The Newton-type scheme converged for all runs (2100), thus confirming the robustness that was stated previously. The mean number of iterations, although it is a function of $\theta_{0} / N_{0}$ and $\theta_{2}$, was never greater than five, leading to $O(M) \log M$ mean floating-point operations per estimate.

The solid lines in Figs. 1 and 3 plot the normalized asymptotic CRB (standard deviation) of parameters $\theta_{1} T_{s}$ and $\theta_{2} T_{s}$ as a function of $\theta_{2} T_{s}$. The curves are parameterized by the SNR. The points inside the circles represent sample standard deviations obtained by running the proposed procedure. Within the uncertainty associated with the sample standard deviation (roughly $0.1 \%$ ), these estimates exhibit a standard deviation that is very close to the asymptotic CRB.

Fig. 2 plots the ratio between the sample variance and the normalized asymptotic CRB ( VAR $_{0}$ ) of $\theta_{1} T_{s}$ resulting from Monte Carlo simulation (100 runs per point) for five different estimators. The

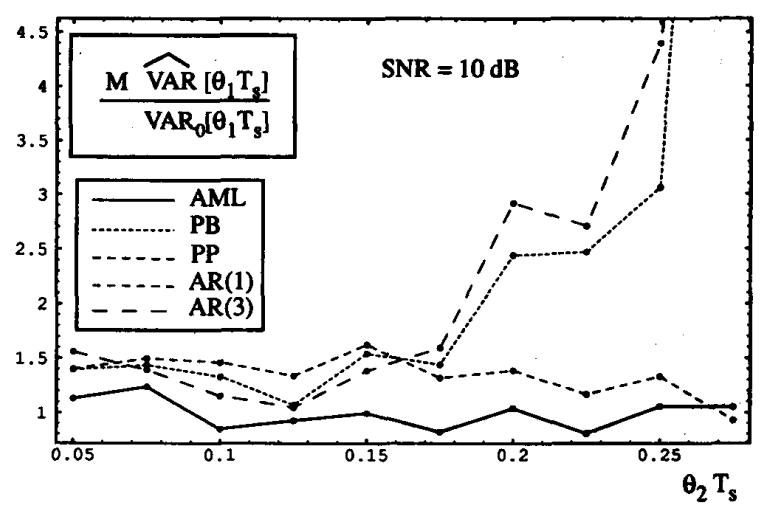

Fig. 2. Ratio between the sample variance ( 100 Monte Carlo runs per point) and the normalized asymptotic CRB (VAR ${ }_{0}$ ) of the first spectral moment (mean frequency) for five different estimators.

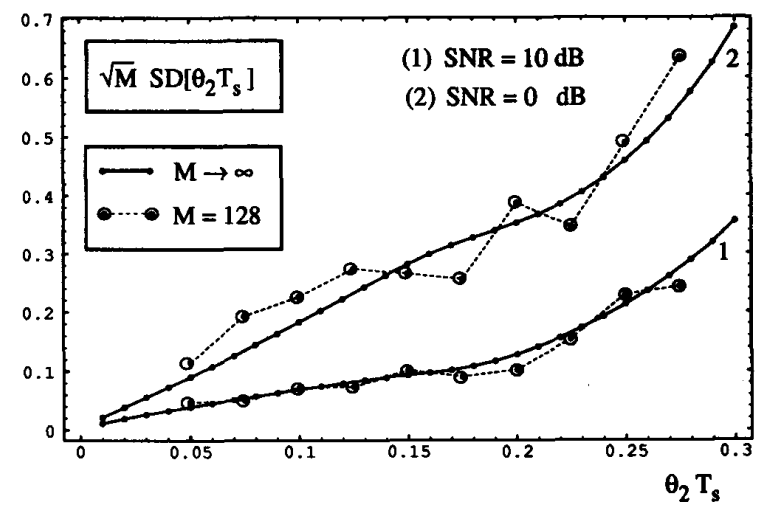

Fig. 3. Solid lines plot the normalized asymptotic CRB (standard deviation) of the second centered spectral moment (spectral width). The points inside the circles show the sample standard deviation (100 Monte Carlo nuns per point) of the AML estimator.

$\operatorname{AR}(i)$ estimator is implemented by applying the SM definition to the spectrum $\hat{S}_{x}$ estimated by assuming an autoregressive process of order $i$. The first thing to note is that the PP and $\operatorname{AR}(1)$ estimators have the same performance (roughly 1.5 times the CRB); this is shown in [3]. The AR(3) and PB estimators seriously degrade their performance for $\theta_{2} T_{s}>0.175$. It is interesting to note that the $\operatorname{AR}(3)$ estimator, compared with the AR(1), yields higher variance for $\theta_{2} T_{s}>0.175$. The justification is that the spectrum associated with the AR(1) estimator is symmetric around $\theta_{1} T_{s}$, whereas this is not true for the AR(3). Therefore, the AR(1) conveys more a priori information about the underlying spectral shape.

Concerning bias, Result 3 led us to the conclusion that $\hat{\theta}_{1}$ is unbiased, whereas $\hat{\theta}_{0}$ and $\hat{\theta}_{2}$ are biased. Fig. 4 plots the relative bias of $\hat{\theta}_{2}$ given by $(10)$ for different SNR's. The points inside the circles show the relative sample bias obtained by Monte Carlo simulations. The accordance between the theoretical curve and the simulation results is evident.

Fig. 5 plots the relative sample bias of $\hat{\theta_{2}} T_{s}$ for SNR equal to 10 $\mathrm{dB}$, drawn from the Monte Carlo simulation, showing that the AML estimator is clearly the best. The PB estimator exhibits two kinds of bias: a) bias due to the mean value of the periodogram, which is the convolution of the true spectrum with a Bartlet window (this bias, which tends asymptotically to zero, affects only the estimates at low spectral widths and can be minimized by convolving the periodogram with an appropriate window); b) bias due to aliasing and independent 


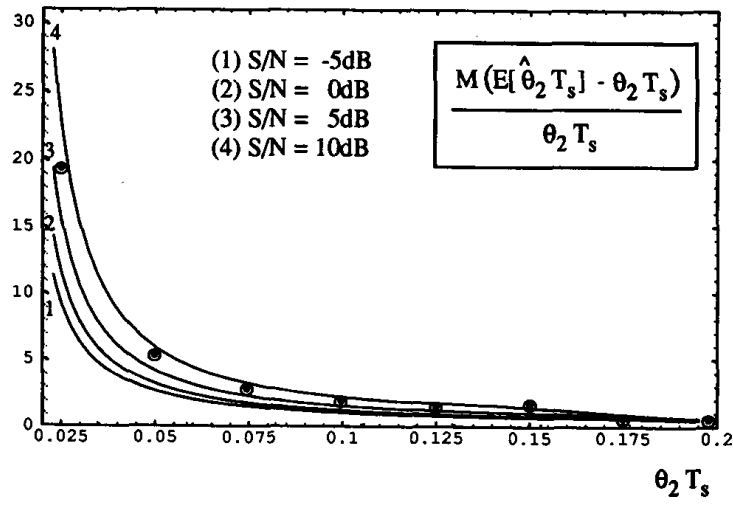

Fig. 4. Solid lines plot the relative bias of the second centered spectral moment (spectral width) according to Result 3 . The points inside the circles represent the sample bias (100 Monte Carlo runs per point) of the AML estimator for SNR equal to $10 \mathrm{~dB}$.

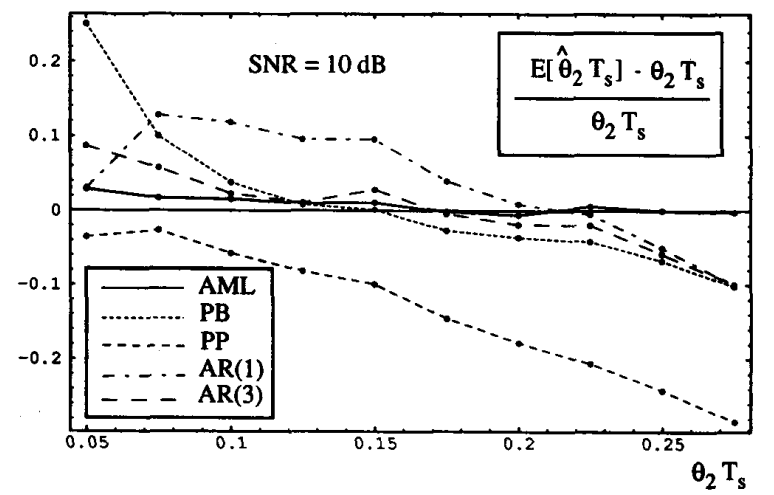

Fig. 5. Relative sample bias ( 100 Monte Carlo runs per point) of the second centered spectral moment (spectral width) for five estimators.

of the sample size. The bias pointed in $b$ ), which can only be avoided by choosing a correct value of $T_{s}$, also affects the $\operatorname{AR}(i)$ estimators regardless of $i$. On the contrary, the AML presents no bias at high SNR, despite the referred aliasing.

\section{CONCLUSION}

This correspondence addressed the estimation of spectral moments of Gaussian processes immersed in Gaussian noise. An estimator exploiting a Whittle's-type objective function, herein termed approximate maximum likelihood (AML), was introduced. Results stating its strong consistency and asymptotical efficiency were presented. A relevant feature of the AML estimator is that it depends on the periodogram, upon which the well-known periodogram-based (PB) estimator is built. This fact was exploited, yielding a Newton-type iterative algorithm initialized with the PB estimate. Furthermore, the Hessian matrix, which is necessary in the Newton-Raphson method, was replaced with the asymptotic Fisher information (which can be computed offline), leading to a highly robust scheme. As a practical example, a Gaussian-shaped spectrum, which is typical of weather radar data, was considered. For a sample size $M=128$, SNR $<10$ $\mathrm{dB}$, and spectral width $\theta_{2} T_{s} \in[0.05,0.3]$, the proposed estimators are nearly unbiased and efficient. Comparisons with the periodogrambased, pulse pair, and autoregressive-based estimators are, in all cases, favorable to the AML.

\section{REFERENCES}

[1] J. B. Dias and J. N. Leitão, "Maximum likelihood estimation of spectral moments at low signal to noise ratios," in Proc. ICASSP '93, Minneapolis, vol. IV, 1993, pp. 149-152.

[2] __ "Nonparametric estimation of mean velocity and spectral width in weather radar," in Proc. IGARSS '95, Florence, 1995.

[3] J. B. Dias, "Estimation of spectral moments: Conceptual and computational aspects," Ph.D. thesis, Instituto Superior Técnico, Lisbon, 1994, (in Portuguese).

[4] D. S. Zrnic, "Estimation of spectral moments for weather echos," IEEE Trans. Geosci. Electron., vol. 17, pp. 113-128, 1979.

[5] K. S. Miller and M. M. Rochewarger, "A covariance approach to spectral moment estimation," IEEE Trans. Inform. Theory, vol. IT-18, pp. 588-596, 1972.

[6] K. Dzhparidze, Parameter Estimation and Hypothesis Testing in Spectral Analysis of Stationary Time Series. New York: Springer-Verlag, 1985.

[7] P. Whittle, "Estimation and information in stationary time series," Arkiv Matematick, vol. 2, pp. 423-434, 1953.

[8] L. L. Scharf, Statistical Signal Processing, Detection, Estimation, and Time Series Analysis. New York: Addison-Wesley, 1991.

[9] J. P. Burg and D. G. Luenberger, "Estimation of structured covariance matrices," Proc. IEEE, vol. 70, pp. 963-974, 1982.

[10] D. B. Williams and D. H. Johnson, "Robust estimation of structured covariance matrices," IEEE Trans. Signal Processing, vol. 41, pp. 2891-2906, 1993.

[11] R. Kumaresan and L. L. Scharf, "An algorithm for pole-zero modeling and spectral analysis," IEEE Trans. Signal Processing, vol. 34, pp. 637-640, 1986.

[12] M. I. Miller and D. L. Snyder, "The role of likelihood and entropy in incomplete data problems: Application to estimating point processes intensity and Toeplitz constrained covariances," Proc. IEEE, vol. 75, pp. 892-907, 1987.

[13] L. H. Janssen and G. A. Van der Spek, "The shape of Doppler spectra from precipitation," IEEE Trans. Aerosp. Electron. Syst., vol. AES-21, pp. 208-219, 1985

\section{Incoherent Receivers in Alpha-Stable Impulsive Noise}

George A. Tsihrintzis and Chrysostomos L. Nikias

Abstract-We compute an incoherent receiver for demodulation of signals with random phase in additive impulsive noise modeled as a bivariate isotropic Cauchy process. Monte-Carlo simulation clearly shows that the proposed Cauchy receiver has significantly improved operating characteristic over the corresponding Gaussian receiver. Moreover, the Cauchy receiver is very robust in the entire class of bivariate isotropic symmetric alpha-stable impulsive noises.

\section{INTRODUCTION}

Communication links are very often corrupted by a clearly nonGaussian interference, termed "impulsive" and characterized by high probability of large amplitudes (e.g., [1]-[5] and [6, ch. 9]). Many natural, as well as man-made, sources of impulsive interference exist, including lightning in the atmosphere, switching transients in power

Manuscript received November 17, 1994; revised March 12, 1995. This work was supported by the Office of Naval Research under contract N0001492-3-1034. The associate editor coordinating the review of this paper and approving it for publication was Roger S. Cheng.

G. A. Tsihrintzis is with the Communications Systems Lab, Department of Electrical Engineering, University of Virginia, Charlottesville, VA 22903 2442 USA.

C. L. Nikias is with the Signal and Image Processing Institute; Department of Electrical Engineering-Systems, University of Southern California, Los Angeles, CA 90089-2564 USA.

IEEE Log Number 9413312. 